\title{
Crioablação da Região Para-Hissiana
}

\author{
Cryoablation of the Para-Hisian region
}

\author{
Leonardo Rezende de Siqueira ${ }^{1, *}$, Nilson Araujo de Oliveira Junior'1, Olga Ferreira de Souza', \\ Rodrigo Periquito Cosenza ${ }^{2}$, Martha Valéria Tavares Pinheiro' ${ }^{1}$, Angelina Camiletti ${ }^{3}$
}

\author{
ORCID IDs \\ Siqueira LR (D) https://orcid.org/0000-0003-1206-0513 \\ Oliveira Junior NA (D) https://orcid.org/0000-0002-9964-6332 \\ Souza OF (D) https://orcid.org/0000-0001-8722-7504
}

\author{
Cosenza RP (iD https://orcid.org/0000-0002-2914-1048 \\ Pinheiro MVT (D) https://orcid.org/0000-0003-1144-9252 \\ Camiletti A (D) https://orcid.org/0000-0002-7867-6794
}

\section{RESUMO}

Fundamentos: A ablação da região para-Hissiana é um desafio devido ao risco de lesão inadvertida do feixe de His. A crioablação, pela sua progressão mais lenta, permite a interrupção da aplicação em caso de sinais de lesões indesejadas e adesividade do cateter durante as aplicações, o que tem tornado a crioablação o método ideal para esses pacientes. Objetivos: Demonstrar os resultados de uma série inicial de pacientes encaminhados para crioablação de vias para-hissianas. Pacientes e métodos: De abril de 2015 a agosto de 2017, 13 pacientes foram encaminhados para crioablação devido à necessidade de abordagem para-hissiana detectada em procedimentos prévios de ablação. Dos 13 pacientes, sete foram submetidos à tentativa de ablação por radiofrequência (RF) e apresentaram insucesso ou recidiva, cinco realizaram apenas estudos eletrofisiológicos, não sendo tentada a ablação, e um foi indicado primariamente. A idade média era $32 \pm 16$ anos. Onze pacientes tinham vias anômalas (VAs) manifestas, um oculta e um taquicardia por reentrada nodal (TRN) com sinais de bloqueio atrioventricular (AV) transitório durante RF. Aplicava-se um ciclo de 4 minutos seguido de mais um ciclo em caso de resultado positivo. Resultados: Dos 13 pacientes, 11 apresentaram sucesso agudo em eliminar a via acessória. Um paciente tinha múltiplas vias acessórias, sendo uma lateral direita e uma lateral esquerda. Nesse paciente foi possível apenas a ablação da via esquerda. Em todos os demais foi observado exuberante potencial hissiano no ponto de aplicação com sucesso. O paciente com TRN foi ablacionado na região $\mathrm{M}$ sem intercorrências. Foram necessárias quatro aplicações em média para eliminação da via acessória com sucesso. A temperatura local média foi de $-74^{\circ} \mathrm{C}$. Em cinco pacientes foi observada a ocorrência de bloqueio do ramo direito (BRD) de terceiro grau. Em um paciente foi interrompida a aplicação precocemente pelo BRD e não fol realizada a aplicação de bônus. Esse foi o único paciente com sucesso agudo que apresentou recidiva clínica. Em nenhum paciente foi observado BAV transitório. Não foram observadas complicações. Conclusão: A crioablação de vias para-hissianas e TRN em regiões mais circunvizinhas do His foi um método eficaz para tratamento nessa população de pacientes refratários ou recusados para tratamento por RF. A ocorrência de BRD agudo não parece um critério para interrupção das aplicações.

PALAVRAS-CHAVE: Crioablação; Para-Hissiana; Vias ântero-septais; Criomapeamento; Crioterapia.

\begin{abstract}
Basis: the ablation of the para-Hisian region is a challenge due to the risk of inadvertent lesion of a bundle of His. Cryoablation, due to its slower progression, allows interruption of the application in case of signs of undesired lesions and catheter adhesion during the applications, which has made cryoablation the ideal method for these patients. Objectives: to demonstrate the results of an initial series of patients referred for cryoablation of para-Hisian pathways. Patients and methods: From April 2015 to August 2017, 13 patients were referred for cryoablation due to the necessity for a para-Hisian approach detected in previous ablation procedures. Of the 13 patients, seven were submitted a radiofrequency ablation attempt (RF) and presented failure or recurrence, five performed only electrophysiological studies, and no ablation was attempted, and one was indicated primarily. The mean age was $32 \pm 16$ years. Eleven patients had manifest anomalous pathways (APS), one hidden and one nodal reentrant tachycardia (NRT) with a transient atrioventricular block (AB) during RF. A cycle of 4 minutes followed by one more cycle in case of a positive result. Results: Of the 13 patients, 11 had an acute success in eliminating the accessory pathway. One patient had multiple accessory pathways, one right side, and one left side. In this patient, it was possible only the ablation of the left pathway. In all others, it was observed exuberant Hisian potential at the point of application with success. The patient with NRT was ablated in the $M$ region without intercurrences. Four applications were required on average to eliminate the accessory pathway successfully. The mean local temperature was $-74{ }^{\circ} \mathrm{C}$. In five patients, the occurrence of third-degree right branch block (RBB) was observed. In one patient, early application of RBB was interrupted and the bonus application was not applied. This was the only acutely successful patient who presented clinical recurrence. Transient AB was not observed in any patient. No complications were observed. Conclusion: Cryoablation of para-Hisian pathways and NRTs in regions surrounding the His was an effective method for treatment in this population of patients refractory or refused for RF treatment. The occurrence of acute RBB does not seem to be a criterion for the interruption of applications.
\end{abstract}

KEYWORDS: Cryoablation; Para-Hisian; Antero-septal pathways; Cryomapping; Cryotherapy.

1.Hospital Universitário Clementino Fraga Filho - Rio de Janeiro/RJ - Brasil.

2.Hospital Federal da Lagoa - Rio de Janeiro/RJ - Brasil.

3. Hospital Quinta D'Or - Rio de Janeiro/RJ - Brasil.

*Autor correspondente: leresiq@gmail.com

Recebido: 15 Abr 2018 | Aceito: 31 Jan 2019

Editor Associado: J. Tarcísio Medeiros de Vasconcelos 


\section{INTRODUÇÃO}

A incidência das vias acessórias foi estimada em três-quatro por 1.000 nascidos vivos. As vias anômalas (VA) ou acessórias são feixes musculares que conectam eletricamente o átrio ao ventrículo ipsilateral, permitindo condução elétrica anormal a despeito da condução nodal atrioventricular (AV) fisiológica. Acredita-se que sejam formadas por falha durante a segmentação embrionária do tubo cardíaco que forma átrios e ventrículos, ocasionando conexão muscular que ultrapassa o anel fibroso valvar. As VAs podem se localizar no anel mitral (65\% dos casos) ou no anel tricúspide. Estima-se que 25\% das vias sejam septais e cerca de $10 \%$ estejam posicionadas em região ântero-septal próxima ao feixe de His. ${ }^{1}$

O tratamento cirúrgico das vias acessórias por cirurgia aberta e posteriormente a ablação por eletrofulguração foram rapidamente substituídos pelo desenvolvimento da ablação por radiofrequência (RF), em 1987. As altas taxas de sucesso e baixo risco de complicações, quando comparadas às técnicas anteriores, promoveram a grande disseminação do método e embasam as diretrizes atuais para tratamento das síndromes de pré-excitação. Os insucessos do procedimento se relacionam, principalmente, com a localização desfavorável da via acessória numa minoria dos pacientes. A localização para-hissiana está relacionada em grandes séries a risco de até $20 \%$ de desenvolvimento de bloqueio VAs total durante a liberação do pulso de $\mathrm{RF}^{2}$

A corrente de RF é uma corrente elétrica alternada de alta frequência unipolar entre o eletrodo distal do cateter de ablação e um eletrodo indiferente de grande superfície posicionado em contato com a pele do paciente. A lesão tecidual se faz pelo aquecimento resistivo do tecido em contato com a ponta do cateter e provavelmente também do efeito elétrico direto. A agressão celular produzida pode se instalar rápida e irreversivelmente, porém tendem a ser mais profunda e mais estável, dependendo do tempo da aplicação, da temperatura e potência atingidas e do contato do cateter com o tecido.

A crioablação foi inicialmente desenvolvida para substituir a ressecção cirúrgica de tumores. Os primeiros cateteres para crioablação de arritmias começaram a ser produzidos na década de 1990. ${ }^{3}$ Atualmente, utilizam-se cateteres com ponta de 4 e de $6 \mathrm{~mm}$ para ablações pontuais e cateteresbalão para isolamento de veias pulmonares.

Para produzir o resfriamento do cateter, nitrogênio líquido é bombeado para dentro do cateter, ocorrendo evaporação interna na sua ponta, o que diminui a temperatura local até $-80^{\circ} \mathrm{C}$. Antes de produzir lesão irreversível, existe a possibilidade de criomapeamento. $\mathrm{O}$ criomapeamento consiste em resfriar o tecido em até $-30^{\circ} \mathrm{C}$ por até 60 segundos, criando uma lesão totalmente reversível. Uma grande vantagem da técnica é o fenômeno de crioadesão, que promove grande estabilidade do cateter quando resfriado, ocorrendo o congelamento do tecido e da ponta do eletrodo na região do contato. ${ }^{4}$

A lesão celular pelo frio é composta por três fases: congelamento, inflamatória e hemorrágica e a de substituição da lesão aguda por fibrose. A fase de congelamento gera morte celular por lesão mitocondrial. A fase inflamatória ocorre nas primeiras 48 horas da aplicação. A fibrose local se instala entre a primeira e a $24^{a}$ semana. A matriz extracelular permanece íntegra e existe pouca ou nenhuma lesão endotelial, o que diminui o risco de tromboembolismo. A lesão pela crioablação não aumenta após o término da aplicação, o que pode ocorrer com a lesão por RF. ${ }^{2}$

As características descritas da ablação por frio rapidamente impulsionaram estudos com ablação de taquicardias por reentrada nodal (TRN), taquicardias mediadas por vias acessórias e fibrilação atrial que revelaram alta taxa de cura e segurança do método, comparáveis à ablação por RF. A utilização para ablação de vias para-hissianas despertou interesse especial pela possibilidade teórica de se evitar lesão irreversível do sistema de condução e aumentar as taxas de sucesso desses procedimentos. Várias séries de casos de ablação por frio de vias para-hissianas têm sido descritas.

Descreve-se, neste artigo, a série inicial consecutiva de pacientes submetidos a crioablação de região para-hissiana.

\section{MÉTODOS}

Série de 13 casos consecutivos de pacientes submetidos à crioablação para vias para-hissianas no período de abril de 2015 a agosto de 2017.

Treze pacientes foram encaminhados para crioablação devido à necessidade de abordagem para-hissiana detectada em procedimentos prévios de ablação.

Dos 13 pacientes, sete foram submetidos à tentativa de ablação por RF e apresentaram insucesso ou recidiva, cinco realizaram apenas estudos eletrofisiológicos, não sendo tentada a ablação, e um foi indicado primariamente à crioablação baseado na localização presumida da via acessória 
fundamentada no eletrocardiograma (ECG). A idade média era de $32 \pm 16$ anos.

Onze pacientes tinham via anômala manifesta préexcitação ventricular ao ECG, um apresentava via anômala oculta e um TRN AV com sinais de bloqueio AV transitório durante tentativa de ablação por $\mathrm{RF}$ em procedimento prévio.

Os procedimentos foram realizados sob sedação guiada por anestesiologista. $\mathrm{O}$ acesso utilizado foi o femoral direito. Aplicava-se um ciclo de crio de 4 minutos seguido de mais um ciclo de 4 minutos em caso de resultado positivo. Em 12 dos 13 pacientes as aplicações foram completas. O tempo de seguimento dos pacientes foi de oito a 36 meses.

\section{RESULTADOS}

As Figs. de 1 a 3 mostram os resultados obtidos. Dos 13 pacientes, 11 apresentaram sucesso agudo em eliminar a via acessória. Um paciente, na verdade, tinha múltiplas vias acessórias, sendo uma lateral direita e uma lateral esquerda. Nesse paciente, foi possível apenas a ablação da via esquerda. Em todos os demais foi observado exuberante potencial hissiano no ponto de aplicação com sucesso.

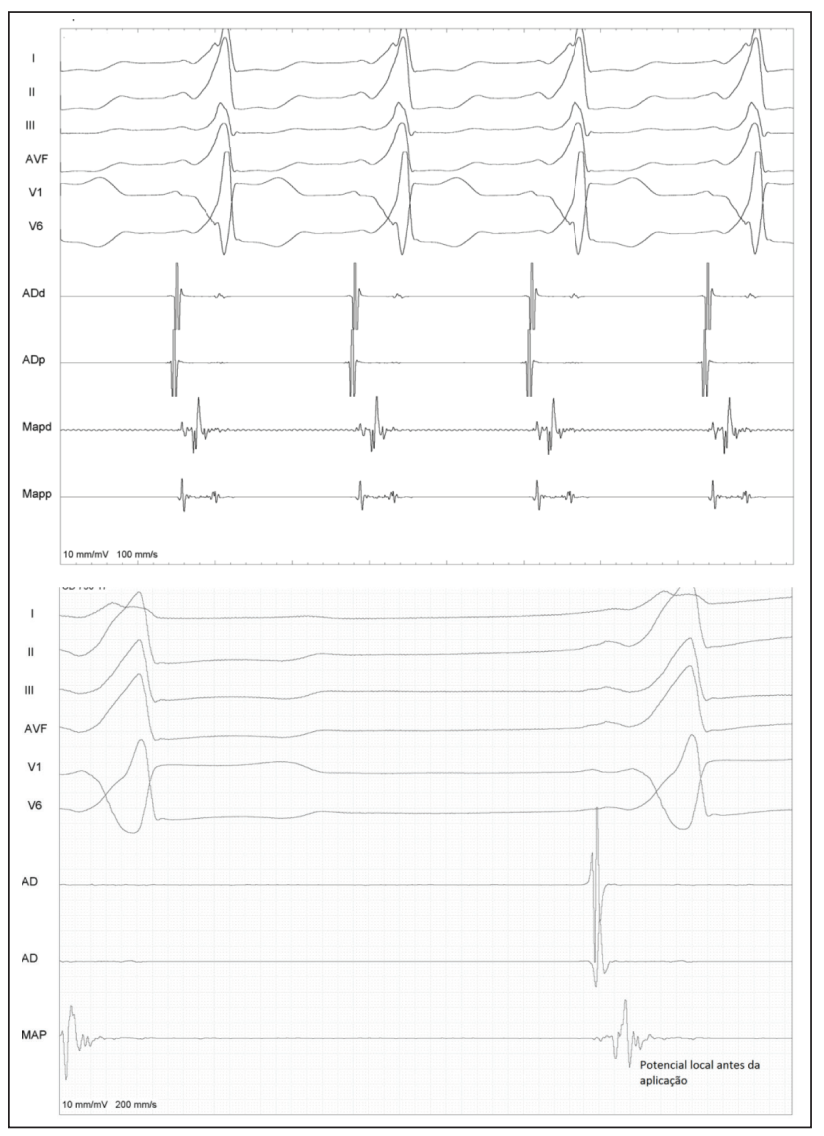

Figura 1. Potencial local anterior a aplicação da crioablação.
O paciente com TRN foi submetido à ablação na região $\mathrm{M}$ sem intercorrências com desaparecimento do salto nodal. Não houve indução de ritmo juncional ativo durante a crioablação. Após a primeira aplicação, identificou-se ablação da via lenta e foi realizada a segunda aplicação de 4 minutos.

Foram necessárias 4 aplicações em média para eliminação da condução pela via acessória. A temperatura local média foi de $-74^{\circ} \mathrm{C}$. Em cinco pacientes, observou-se a ocorrência de bloqueio do ramo direito (BRD) de terceiro grau durante a aplicação.

A duração média dos procedimentos foi de 52 minutos. O tempo médio de escopia foi de 6 minutos.

Em um paciente, interrompeu-se a aplicação precocemente pelo $\mathrm{BRD}$ (130 segundos) e foi não realizada a aplicação de reforço. Observou-se, em consulta 14 dias após o procedimento, o retorno da pré-excitação ventricular. Esse foi o único paciente com sucesso agudo que apresentou recidiva da pré-excitação ou de retorno dos sintomas de palpitação.

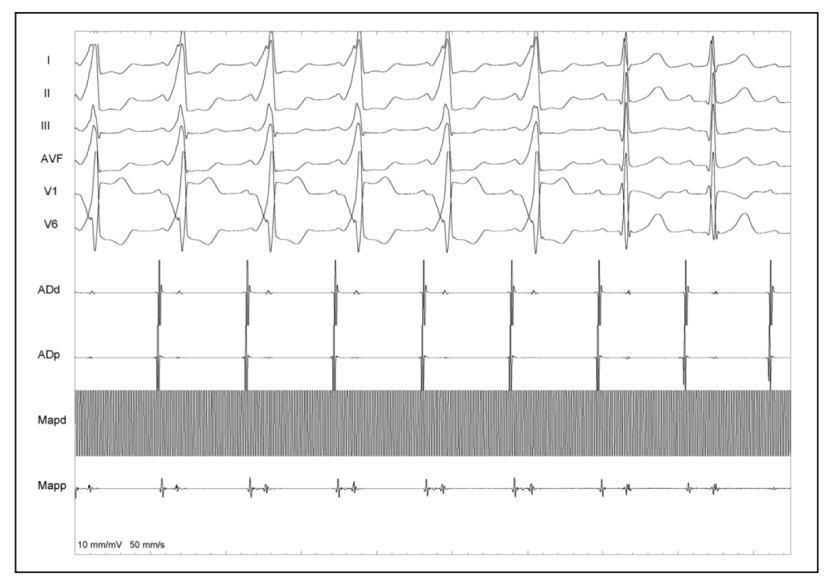

Figura 2. Desaparecimento da pré-excitação durante a crioablação.

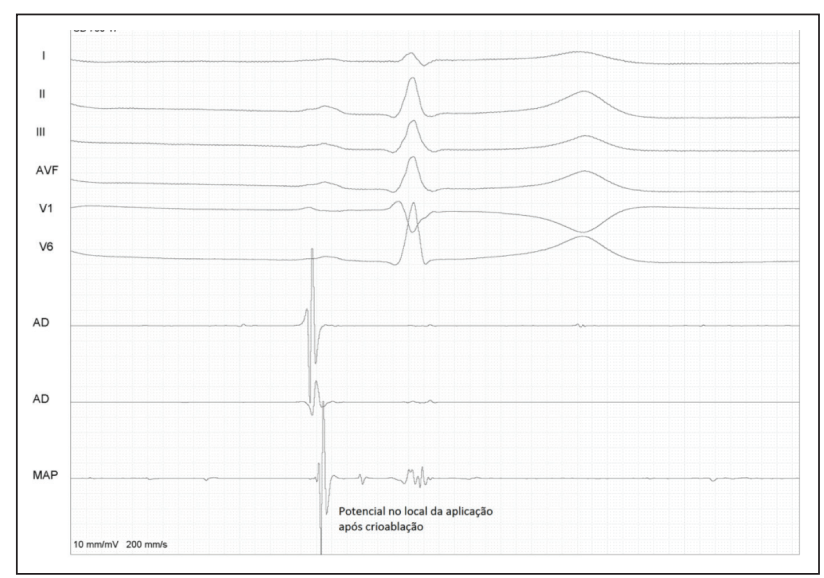

Figura 3. Potencial de His no local da crioablação após desaparecimento da pré-excitação ventricular. 
Não foi observado bloqueio AV transitório de qualquer grau em nenhum paciente. As medidas dos intervalos HV não revelaram valores anormais após as aplicações. Não foram observadas complicações a curto e médio prazos. Os pacientes não apresentaram recidiva de taquicardia ou pré-excitação ventricular ou bloqueio AV no seguimento.

\section{DISCUSSÃO}

As vias acessórias para-hissianas sempre foram um desafio para a eletrofisiologia na era da ablação por RF devido ao risco de lesão irreversível do nodo AV durante a liberação do pulso de $R F$. A lesão do nodo $A V$ pode se instalar rapidamente sem sinais de aviso premonitórios.

Diversas técnicas de mapeamento e ablação por RF foram descritas na tentativa de aumentar o sucesso da ablação e diminuir o risco do procedimento nas vias ântero-septais direitas. Mapeamento e ablação de cúspide coronariana direita, uso de mapeamento eletroanatômico e liberação de energia com baixa potência, abordagem por acesso superior (jugular ou subclávia) e utilização de sistema de navegação magnética foram publicados em relatos de casos.
O procedimento da crioablação guarda grande similaridade com o procedimento de ablação por RF em relação à via de acesso, sedação e uso de radioscopia. $\mathrm{O}$ manuseio do cateter de crioablação é muito semelhante ao do cateter de RF, o que torna a técnica de fácil execução para eletrofisiologistas habilitados.

A crioablação tem se mostrado método eficaz e seguro em arritmias nas quais a região a ser lesionada se encontra contígua ao feixe de His. A técnica se mostrou segura mesmo em crianças e com altas taxas de cura a longo prazo em diversas séries de pacientes relatadas na literatura. O procedimento de crioablação é semelhante ao padrão de ablação por RF no que se refere ao acesso venoso femoral e à utilização de radioscopia.

Não existem estudos comparativos diretos publicados de ablação por RF e crioablação. Nossa série inicial de casos mostra alta taxa de sucesso e segurança da crioablação em vias acessórias para-hissianas em conformidade com a literatura atual.

\section{CONTRIBUIÇÃO DOS AUTORES}

Todos os autores contribuiram igualmente para este artigo.

\section{REFERÊNCIAS}

1. Yildirim I, KaragözT, Ertuğrul I, KaragözAH. Özer S. Efficacy and safety of cryoablation of parahissian accessory pathways in children: A Single Institution Study. Pacing Clin Electrophysiol. 2013;36(12)1495-502. https://doi.org/10.1111/pace.12268

2. Cay S, Aras D, Topaloglu S, Ozcan F, Ozeke O. Various routes and techniques for ablation of parahisian bypass tracts. Int J Cardiol. 2016;223:217. https://doi.org/10.1016/j.ijcard.2016.08.257
3. Liao Z, Zhan X, Wu S. Successful radiofrequency ablation of a parahisian accessory pathway from the right coronary cusp. Int J Cardiol. 2015;186:41-2. https://doi.org/10.1016/ j.ijcard.2015.03.231

4. Zeljko HM, Yue A. Europace. 2015;17(11):1707. https://doi. org/10.1093/europace/euv254 